\title{
Utilisation du froid pour la stérilisation des viandes ladres à l'abattoir frigorifique de Fort-Lamy
}

\author{
par M. GRABER, M. TROUETTE et A. CHAILLOUX \\ (avec la collaboration technique de $\mathrm{M}^{\text {ney }}$ BRUNET et BROK)
}

\begin{abstract}
RESUME
Les auteurs opéraient sur des carcasses d'animaux atteints de cysticercose, originaires des régions sahélo-soudaniennes du Tchad, d'un poids moyen de $187 \mathrm{~kg}$ pour les zébus et de $60 \mathrm{~kg}$ pour les porcs. Ils ont obtenu leur complète stérilisatión en les entreposant, après réfrigération à $+5^{\circ} \mathrm{C}$, à une température de $-15^{\circ} \mathrm{C}$, pendant une durée de 54 heures pour les zébus et 35 heures pour les porcs.

Ils fixent ainsi les normes de sterilisation des carcasses ladres applicables en général dans la plupart des régions d'Afrique à élevage extensif.
\end{abstract}

\section{INTRODUCTION}

Il existe plusieurs façons d'assainir les vaindes ladres : la chaleur, le salage, la mise en saumure, l'irradiation par les rayons $X$ (PAWEL et JANICEK, 1963). Chacune a ses avantages et ses inconvénients : la chaleur altère les qualités organoleptiques de la viande, le salage et la saumure n'ont que des applications limitées et l'irradiation n'est guère entrée dans les mœurs, car la législation sanitaire d'un grand nombre de pays interdit, pour l'instant, la commercialisation des carcasses ainsi traitées.

Le froid est utilisé depuis le début du siècle. Si la réfrigération simple (de $0^{\circ} \mathrm{C}$ à $+4^{\circ} \mathrm{C}$ ) est à proscrire (EUZEBY, 1966), la congélation, par contre, présente de gros avantages et ses modalités ont fait l'objet de très longues discussions (VILJOEN, 1937). Finalement, de

(*) Article publié in: Bull. Inst. int. Froid, 1970, 50 (3): $559-70$.

Institut d'Elevage et de Médecine Vétérinaire des Pays Tropicaux. Laboratoire de Farcha, Fort-Lamy (République du Tchad). Abattoir Frigorifique de Farcha. nombreux pays, dont la France (C.M. du 16 janvier 1964), ont adopté les normes suivantes: maintien des carcasses en frigorifique 10 jours à $-10^{\circ} \mathrm{C}$. En Allemagne, certains auteurs mettent la viande préalablement réfrigérée à $0^{\circ} \mathrm{C}, 144$ heures (BRAND, 1961) ou 134 heures (BARTELS et TANDLER, 1961) à $-10^{\circ} \mathrm{C}$. La température à cœur doit rester à $-3^{\circ} \mathrm{C}$ durant 24 heures au moins.

C'est là une opération qui exige du temps et de vastes locaux.

Depuis quelques années, on s'est efforcé de rechercher d'autres procédés plus rapides. La congélation est à l'ordre du jour (LACROUTS, 1964) et a fait l'objet de plusieurs études (MONZINI et LANDI, 1954; BARTELS et TANDLER, 1961 et 1963; BIAGI et collab., 1965; MALHEIRO et collab., 1966). L'une d'entre elles concerne l'abattoir de Bangui (R.C.A.) où la viande ladre est soumise à une température de $-15^{\circ} \mathrm{C}$ à cour durant 24 heures (LAURENT et DAMARY, 1964). avec plein succès, semble-t-il.

Cependant, les résultats varient selon les espèces, les régimes et les expérimentateurs et 
il faut bien reconnaître qu'il n'y a pas actuellement de technique standard pour stériliser les viandes parasitées (LACROUTS, 1964).

Aussi nous a-t-il paru intéressant de rechercher et de mettre au point une méthode adaptée aux conditions propres à la République du Tchad: c'est l'objet du présent travail qui a été réalisé en collaboration étroite entre la section d'Helminthologie du Laboratoire de Farcha et l'abattoir frigorifique de Fort-Lamy. Il est probable que les résultats de cette étude pourront trouver leur application dans d'autres pays africains où l'élevage bovin est pratiqué de la même façon avec des taux de cysticercose peu différents.

\section{LA CYSTICERCOSE A L'ABATTOIR DE FORT-LAMY}

L'abattoir de Fort-Lamy sacrifie, selon les années, de 35 à 57.000 zébus et de 1.000 à 1.400 porcs. La viande ainsi produite est destinée à l'exportation avion et à la consommation locale.

La cysticercose bovine à Cysticercus bovis et la cysticercose porcine à Cysticercus cellulosae sont également répandues, comme l'indique le tableau $n^{n} 1$ qui concerne les zébus adultes et les porcs.

TABLEAU $\mathrm{N}^{\circ} \mathrm{I}$

Pourcentage d'animaux trouvés porteurs de Cysticerques

à l'abattoir de Fort-Lamy.

\begin{tabular}{|c|c|c|}
\hline Années & Zébus adu1tes & Porcs \\
\hline $1952-1959$ & $0,4-0,6 \mathrm{p} \cdot 100$ & - \\
\hline $1960-1963$ & $1,2-1,67 \mathrm{p} \cdot 100(1961)$ & - \\
\hline $1964-1969$ & $\begin{array}{c}1,46 \mathrm{p} \cdot 100 \\
\text { (sur } 210.654 \text { têtes) }\end{array}$ & $\begin{array}{c}6,94 \mathrm{p} .100 \\
\text { (sur } 6.106 \text { têtes) }\end{array}$ \\
\hline
\end{tabular}

Ces derniers sont peu nombreux (environ 6.000) au Tchad et élevés surtout dans les Préfectures du Sud (Mayo-Kebbi, Logone, Tandjilé et Moyen-Chari). L'apparition des premiers cas de cysticercose porcine semble avoir pour origine des achats effectués dans certaines zones de Mayo-Kebbi. Dans cette espèce, la ladrerie est souvent généralisée et justifie la saisie totale.

Quant à la cysticercose, elle est enzootique au Tchad. Une enquête précise faite au Laboratoire de Farcha et portant sur 4.015 têtes donne une moyenne nationale de 8,56 p. 100 , avec de sérieux écarts selon les régions. L'Ouest et le Nord-Ouest du pays sont beaucoup moins touchés. Or, la source d'approvisionnement principale de l'abattoir est le Kanem où le taux d'infestation ne semble pas dépasser 4,9 p. 100 (GRABER, 1968).

La distorsion observée entre la moyenne nationale et la moyenne de l'abattoir peut également être expliquée (GRABER et THOME, 1964):
- par le fait que plus du tiers des animaux abattus appartenaient à de grands nomades, Bororos notamment, dont le cheptel héberge 4 fois moins de cysticerques que le bétail sédentaire (GRABER et TABO, 1968);

- par les techniques d'inspection. Le parasitologue, au cours de ses enquêtes, est obligé de mettre l'animal suspect en menus morceaux. Certaines localisations profondes (Anconés, muscles de la cuisse), fréquentes en Afrique centrale (GRABER, 1959), apparaissent alors nettement. A l'abattoir, il n'est pas possible d'opérer de cette manière: outre l'opposition des bouchers, les demi-carcasses réfrigérées qui sont destinées à voyager par avion sur de longues distances doivent subir le minimum de manipulations et demeurer entières, ce qui exclut la "levée de l'épaule" et les incisions au niveau des muscles adducteurs de la cuisse : en milieu tropical, on risque, en effet, de provoquer des contaminations bactériennes sévères et rapides par développement de germes dans le suc musculaire exsudé. Il faut l'éviter à tout 
prix, sinon la viande sera saisie à l'aérodrome d'arrivée.

Le nombre de cysticerques rencontrés à l'inspection est variable. A la coupe, sont décelées, en général, une ou deux vésicules, souvent calcifiées ou en cours de calcification et localisées à la langue. Dans ce cas, s'il n'y a pas d'autres parasites dans les muscles, l'abat seul est saisi et la viande remise en circulation. La cysticercose généralisée (tableau $n^{\circ} 2$ ) est rare et ne donne pas lieu à stérilisation : l'animal atteint est brûlé à l'incinérateur ou transformé en poudre. L'assainissement ne touche donc que des zébus moyennement parasités (de 5 à 20 cysticerques), soit environ 0,19 p. 100 des 245.117 bêtes tuées de 1963 à 1969. Selon les années et les saisons, leur nombre est sujet à d'amples fluctuations (tableau $\mathbf{n}^{\circ} 3$ ). Le maximum atteint a été de 41 en octobre 1968.

Il est bon de préciser qu'avant l'emploi du froid, les carcasses ladres étaient traitées par la chaleur et soumises à une cuisson prolongée, ce qui diminuait la valeur de la viande et posait un problème d'écoulement sur les marchés locaux.

TABLEAU $\mathrm{N}^{\bullet}$ II

Cysticercose bovine. Nombre de bêtes saistes cotalement et nombre de bêtes assalnies

\begin{tabular}{|c|c|c|c|}
\hline Années & $\begin{array}{c}\text { Cysticercose généralisée } \\
\text { (salaies tatales) }\end{array}$ & $\begin{array}{l}\text { Cysticercose moyenne } \\
\text { (assainissement) }\end{array}$ & $\begin{array}{c}\text { Nombre } d^{\dagger} \text { an } 1 \text { maux } \\
\text { abattus }\end{array}$ \\
\hline 1963 & 13 & 60 & 34.463 \\
\hline 1964 & 8 & 86 & 37.056 \\
\hline 1965 & 22 & 66 & 38.111 \\
\hline 1966 & 24 & 74 & 36.143 \\
\hline 1967 & 8 & 67 & 42.560 \\
\hline 1968 & 11 & 116 & 56.784 \\
\hline Total & 86, solt 0,03 p. 100 & 469 , solt 0,19 p. 100 & 245.117 \\
\hline
\end{tabular}

TABLEAU $\mathrm{N}^{\bullet}$ III

Nombre de zébus adultes ladres assainis à 1'abattolr de Fort-Lamy de 1963 à 1966.

\begin{tabular}{|c|c|c|c|c|c|c|}
\hline \multirow{2}{*}{$M \circ 1 \mathrm{~s}$} & \multicolumn{6}{|c|}{ A n n é } \\
\hline & 1963 & 1964 & 1965 & 1966 & 1967 & 1968 \\
\hline Janvier & 5 & 6 & 9 & 4 & 6 & 6 \\
\hline Février & 4 & 3 & 5 & 4 & 8 & 0 \\
\hline Mars & 4 & 6 & 4 & 7 & 6 & 2 \\
\hline Avril & - & 2 & 6 & 8 & 3 & 1 \\
\hline Ma1 & 1 & 1 & 1 & 6 & 7 & 3 \\
\hline Juln & 4 & 1 & 4 & 2 & 14 & 2 \\
\hline Jut1let & 7 & 12 & 3 & 12 & 1 & 7 \\
\hline Août & 11 & 10 & 4 & 6 & 7 & 4 \\
\hline Seftembre & 8 & 6 & 6 & 2 & $B$ & 20 \\
\hline Octobre & 9 & 13 & 11 & 4 & 2 & 41 \\
\hline Novembre & 7 & 16 & 7 & 12 & 3 & 16 \\
\hline Décembre & - & 10 & 6 & 7 & 2 & 13 \\
\hline Iotal & 60 & 86 & 66 & 74 & 67 & 116 \\
\hline
\end{tabular}




\section{MATERIEL ET METHODE}

\section{Les animaux}

Au total, 45 porcs et 23 zébus Arabes ou Bororos ladres ont été utilisés, dont 17 porcs et 9 zébus pour les essais proprement dits.

Ils étaient tous atteints de cysticercose massive généralisée et normalement justiciables d'une saisie totale suivie d'une destruction à l'incinérateur.

Les poids variaient entre 48 et $75 \mathrm{~kg}$ pour les porcs (en moyenne $60,750 \mathrm{~kg}$ ) et entre 117 et $250 \mathrm{~kg}$ pour les boufs (en moyenne $186,5 \mathrm{~kg}$ ).

Dans l'ensemble, il s'agissait de carcasses en excellent état et dont la graisse de couverture était très abondante.

\section{L'installation}

Compte tenu de ce qui a été dit plus haut, il était nécessaire d'avoir à sa disposition un tunnel $\left.{ }^{(}\right)$de congélation adapté au volume moyen de viande à stériliser et permettant de faire face à toutes les situations, notamment à celle créée, certains mois, par l'existence d'un nombre anormalement élevé d'animaux ladres.

Il a été obtenu par transformation d'un local, servant précédemment à la réfrigération, dont le plafond a été abaissé : la hauteur est de $2,20 \mathrm{~m}$. Un sas a été construit. L'isolement thermique est réalisé au moyen de Frigolit sous une épaisseur de $18 \mathrm{~cm}$. Le volume total est de $15 \mathrm{~m}^{3}$ et permet d'entreposer :

- 15 porcs de $50 \mathrm{~kg}$, soit $750 \mathrm{~kg}$;

- 4 bœufs de $185 \mathrm{~kg}$, soit $740 \mathrm{~kg}$.

Le tunnel de congélation a reçu l'équipement suivant :

- Un groupe frigorifique Satam-Nevé de 7.900 frigories/heures à 600 tours-minutes.

- Un moteur électrique Drouard $5 \mathrm{CV}$ triphasé $220 / 380$.

- Un évaporateur.

:- Deux ventilateurs.

L Un détendeur.

:- Des appareils de contrôle H.P.B.P.

(1) -Il s'agit d'un procédé de congélation semi-rapide.
Les premiers essais ont été entrepris à partir de viandes chaudes, tout au moins pour les porcs. Techniquement, ce procédé est fortement déconseillé, sinon interdit, car la viande, après décongélation, est humide, mouillée et grise. Dans le cas présent, cette technique n'a été utilisée que dans un but purement expérimental (étude du comportement et de la survie des cysticerques in situ).

\section{Technique}

Les animaux ladres sont retirés de la circulation et numérotés. On prélève en différents points (en surface et en profondeur) le plus grand nombre possible de cysticerques et on s'assure de leur vitalité. De nombreuses méthodes ont été décrites (VILJOEN, 1937). La plus simple consiste à placer les vésicules, préalablement ouvertes, dans de la bile de bouf ou de porc selon l'espèce en cause, bile maintenue à la température de $39^{\circ} \mathrm{C}$ dans une étuve adéquate. Le cysticerque vivant s'évagine au bout d'un temps variable et présente des mouvements facilement décelables à la loupe. Les observations, faites de 5 heures en 5 heures, durent 24 heures au minimum. Cette méthode, facile à mettre en cuvre, donne au Laboratoire de Farcha toute satisfaction depuis 15 ans.

Après congélation, la même opération est répétée sur les cysticerques de surface et de profondeur (surtout au niveau de la cuisse). Dans l'un et l'autre cas, le nombre de Cestodes larvaires testés est supérieur à 10 .

L'appréciation de la température "à cour" se fait à l'aide d'une sonde placée dans les masses musculaires les plus profondes (le globe) au contact de l'os - la courbe thermique est enregistrée automatiquement sur un tambour placé à l'extérieur du tunnel.

\section{RESULTATS}

\section{Résultats expérimentaux}

Les quartiers $\left({ }^{2}\right)$ sont pendus dans la pièce maintenue à $-14^{\circ} \mathrm{C}$. La courbe obtenue a l'allure indiquée au graphique $\mathrm{n}^{*} 1$. Après un temps variable, selon qu'il s'agit de viandes réfrigérées ou de viandes chaudes, on atteint un palier qui, dans les conditions de l'expé-

(2) Bœuf seulement, les porcs sont entiers. 
Grophique $n^{\circ} 1$ : Courbes de congélation - ambiance $-14^{\circ} \mathrm{C}$

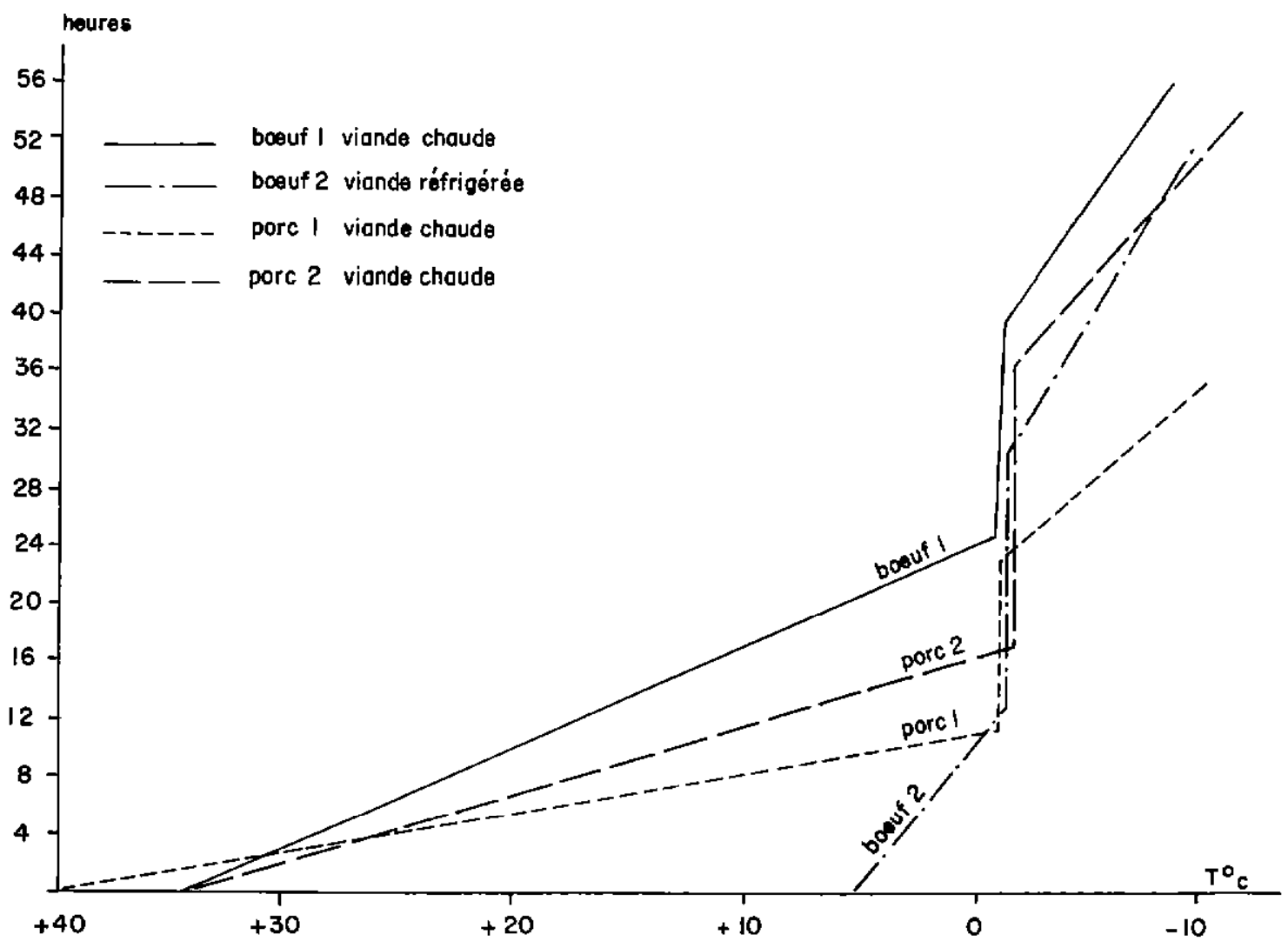

TABLEAU $\mathrm{N}^{\circ}$ IV

Stêrilisation de viandes ladres de zëbus. Rêsultats expérımentaux.

\begin{tabular}{|c|c|c|c|c|c|c|c|c|c|}
\hline Zébu $n^{\bullet}$ & 1 & 2 & 3 & 4 & 6 & 8 & 12 & 9 & 13 \\
\hline Température initiale* & $+8^{*}$ & $+3^{*}$ & $+9^{\circ}$ & $+4^{*}$ & $+5^{\circ}$ & $+2^{\bullet}$ & $+5^{\circ}$ & $+34^{\circ}$ & $+B^{*}$ \\
\hline Amblance & $-18^{*}$ & $-16^{\circ}$ & $-14^{*}$ & $-14^{\circ}$ & $-14^{\circ}$ & $-14^{\bullet}$ & $-14^{\circ}$ & $-14^{\circ}$ & $-14^{\circ}$ \\
\hline $\begin{array}{l}\text { Temips mis pour at- } \\
\text { teindre }-1,5^{\circ} \mathrm{C}\end{array}$ & $14 \mathrm{~h}$ & $35 \mathrm{~h}$ & $24 \mathrm{~h}$ & $22 \mathrm{~h}$ & $12 \mathrm{~h}$ & $12 \mathrm{~h}$ & 12 h 30 & $24 \mathrm{~h}$ & \\
\hline $\begin{array}{l}\text { Durée du palfer } \\
\text { de congëlation }\end{array}$ & $19 \mathrm{~h}$ & $19 \mathrm{~h}$ & $13 \mathrm{~h}$ & $15 \mathrm{~h}$ & $18 \mathrm{~h}$ & $19 \mathrm{~h}$ & $15 \mathrm{~h}$ & $15 \mathrm{~h}$ & \\
\hline Température finale * & $-16^{*}$ & $-14^{\circ}$ & $-12^{*}$ & $-12^{\circ}$ & $-10^{\circ}$ & $-8^{\circ}$ & $-5^{a}$ & $-8^{\circ}$ & $-7^{\circ}$ \\
\hline $\begin{array}{l}\text { Temps mis pour 1'ob- } \\
\text { tenir }\end{array}$ & $35 \mathrm{~h}$ & $17 \mathrm{~h}$ & $21 \mathrm{~h}$ & $1^{9 h}$ & $22 \mathrm{~h}$ & $17 \mathrm{~h}$ & $17 \mathrm{~b}$ & $15 \mathrm{~h}$ & \\
\hline $\begin{array}{l}\text { Durée totale de } \\
\text { l'opération }\end{array}$ & $6 \mathrm{~B} \mathrm{~h}$ & $71 \mathrm{~h}$ & $58 \mathrm{~h}$ & $56 \mathrm{~h}$ & $52 \mathrm{~h}$ & $48 \mathrm{~h}$ & $44 \mathrm{~h} 30$ & $54 \mathrm{~h}$ & $50 \mathrm{~h} 30$ \\
\hline $\begin{array}{l}\text { Survie des } \\
\text { cysticerques }\end{array}$ & $\begin{array}{l}\text { tous } \\
\text { morts }\end{array}$ & $\begin{array}{l}\text { tous } \\
\text { morts }\end{array}$ & $\begin{array}{l}\text { tous } \\
\text { morts }\end{array}$ & $\begin{array}{l}\text { tcus } \\
\text { morts }\end{array}$ & $\begin{array}{l}\text { tous } \\
\text { morts }\end{array}$ & $\begin{array}{l}\text { tous } \\
\text { morts }\end{array}$ & $\begin{array}{r}\text { vivants } \\
4,6 p .100\end{array}$ & $\begin{array}{l}\text { tous } \\
\text { morts }\end{array}$ & $\begin{array}{l}\text { vivants } \\
3,5 \text { p. } 100\end{array}$ \\
\hline Poids (en kg) & 220 & 150 & 205 & 130 & 148 & 196 & 188 & & 158 \\
\hline
\end{tabular}

* Température à coeur en degrés centigrades. 
TABLEAU $\mathrm{N}^{*} \mathrm{~V}$

Stêrilisation de porcs ladres. Résultats expérimentaux.

\begin{tabular}{|c|c|c|c|c|c|c|c|c|c|c|c|c|c|}
\hline \multicolumn{8}{|c|}{$\mathrm{A}$ mb i ance $-14^{\circ} \mathrm{C}$} & \multicolumn{6}{|c|}{ A m b i a n c e $-15^{\circ} \mathrm{C}$} \\
\hline$P \circ \Gamma \mathrm{c} \mathrm{n}^{0}$ & $1-2-3$ & 5 & 7 & 10 & $11-12-13$ & 14 & 15 & 1 & 2 & 3 & 4 & 5 & 6 \\
\hline Tempêrature initiale * & $36^{\circ}$ & $34^{\circ}$ & $39^{\circ}$ & $3^{\circ}$ & $36^{\circ}$ & $34^{\circ}$ & $34^{\circ}$ & $36^{\circ}$ & $34^{\circ}$ & $38^{\circ}$ & $36^{*}$ & $34^{\circ}$ & $35^{*}$ \\
\hline $\begin{array}{l}\text { Temps mis pour at- } \\
\text { teindre }-1,5^{\circ} \mathrm{c}\end{array}$ & $23 \mathrm{~h}$ & $17 \mathrm{~h}$ & $11 \mathrm{~h}$ & $6 \mathrm{~h}$ & $23 \mathrm{~h}$ & - & - & - & - & - & - & - & - \\
\hline $\begin{array}{l}\text { Durée du palier de } \\
\text { congêlation }\end{array}$ & $12 \mathrm{~h}$ & $20 \mathrm{~h}$ & $11 \mathrm{~h}$ & $10 \mathrm{~h}$ & $12 \mathrm{~h}$ & - & - & - & - & - & - & - & - \\
\hline Tempêrature finale* & $-4^{\circ}$ & $-12^{\circ}$ & $-10^{\circ}$ & $-6^{\circ}$ & $-4^{\circ}$ & $-8^{\circ}$ & $-8^{\bullet}$ & - & - & - & - & - & - \\
\hline Temps mis pour l'obtenir & $14 \mathrm{~h}$ & $17 \mathrm{~h}$ & $12 \mathrm{~h}$ & $12 \mathrm{~h}$ & $14 \mathrm{~h}$ & - & - & - & - & - & - & - & - \\
\hline $\begin{array}{l}\text { Durée totale de } \\
1^{\prime} \text { opération }\end{array}$ & $49 \mathrm{~h}$ & $54 \mathrm{~h}$ & $34 \mathrm{~h}$ & $28 \mathrm{~h}$ & $49 \mathrm{~h}$ & 33 h 30 & $38 \mathrm{~h}$ & $30 \mathrm{~h}$ & $30 \mathrm{~h}$ & $30 \mathrm{~h}$ & $30 \mathrm{~h}$ & $30 \mathrm{~h}$ & $30 \mathrm{~h}$ \\
\hline $\begin{array}{l}\text { Survie des cysticer- } \\
\text { ques }\end{array}$ & $\begin{array}{l}\text { vivants } \\
17 p .100\end{array}$ & $\begin{array}{l}\text { tous } \\
\text { morts }\end{array}$ & $\begin{array}{l}\text { tous } \\
\text { morts }\end{array}$ & $\begin{array}{l}\text { tous } \\
\text { morts }\end{array}$ & $\begin{array}{l}\text { vivants } \\
13 \text { p.100 }\end{array}$ & $\begin{array}{l}\text { vivants } \\
26 \mathrm{p} .100\end{array}$ & $\begin{array}{l}\text { tous } \\
\text { morts }\end{array}$ & $\begin{array}{l}\text { tous } \\
\text { morts }\end{array}$ & $\begin{array}{l}\text { tous } \\
\text { morts }\end{array}$ & $\begin{array}{r}\text { tous } \\
\text { morts }\end{array}$ & $\begin{array}{l}\text { vivants } \\
30 p .100\end{array}$ & $\begin{array}{l}\text { tous } \\
\text { morts }\end{array}$ & $\begin{array}{r}\text { tous } \\
\text { morts }\end{array}$ \\
\hline Poids (en kg) & $\begin{array}{l}58 \\
\text { (moy.) }\end{array}$ & 62 & 48 & 58 & - & 64 & 59 & 61 & 67 & 64 & 72 & 61 & 60 \\
\hline
\end{tabular}

* Tempêrature à coeur en degrếs centigrades 
rience, est de $-1,5^{\circ} \mathrm{C}$ à cour. Durant quelques heures, cette température ne subit pas de modifications: c'est le palier de congélation qui est suivi d'un refroidissement accéléré. On arrête l'expérience quand la température à cœur atteint le degré voulu.

Après contrôle de la survie des cysticerques, on calcule la durée totale de l'expérience.

Les résultats figurent aux tableaux 5 et 6 .

1.1. Chez le zébu, il reste encore des cysticerques vivants en petit nombre à $-5^{\circ},-7^{\circ} \mathrm{C}$ à cour. A partir de $-8^{\circ} \mathrm{C}$, la stérilisation est totale, ce qui correspond, dans les conditions propres à l'expérience et pour des carcasses réfrigérées, entre $+4^{\circ}-5^{\circ} \mathrm{C}$ à 54 heures d'entreposage dans une ambiance de $-14^{\circ} \mathrm{C}$.

Cependant, il faut tenir compte de plusieurs facteurs qui risquent de fausser les résultats :

- le poids des carcasses et leur conformation qui ne sont pas les mêmes, selon que lion s'adresse à des zébus arabes ou à des zébus bororos plus lourds et mieux conformés;

- l'état d'engraissement. La conductibilité de la graisse est inférieure d'environ 35 p. 100 à celle du muscle (KALLER, 1931). Comme nous avons en général opéré sur des carcasses dont la graisse de couverture était abondante, la durée d'entreposage dans le tunnel de congélation à $-14^{\circ} \mathrm{C}$ (après réfrigération) pourra être diminuée de 3 p. 100 environ, lorsqu'il s'agira de traiter des carcasses maigres.

Pour plus de sécurité, pour un même temps d'exposition, la température a été abaissée de $-1^{\circ} \mathrm{C}$; les normes retenues pour le zébu sont alors de 54 heures à $-15^{\circ} \mathrm{C}$.

1.2. Chez le porc, dont le poids moyen s'établit autour de $58 \mathrm{~kg}$, les résultats sont irréguliers entre $-4^{\circ} \mathrm{C}$ et $-8^{\circ} \mathrm{C}$ à cour. En viande chaude $\left({ }^{9}\right)$, il faut, entre $-8^{\circ} \mathrm{C}$ et

(3) En général, les carcasses de porc sont réfrigérées à $+1^{\circ} \mathrm{C}$ et même à $0^{\circ} \mathrm{C}$.

TABLEAU $\mathrm{N}^{\mathrm{B}} \mathrm{VI}$

Applications pratiques chez le porc.

Stérilisation après entreposage durant 35 heures dans une ambiance de $-15^{\circ} \mathrm{C}$.

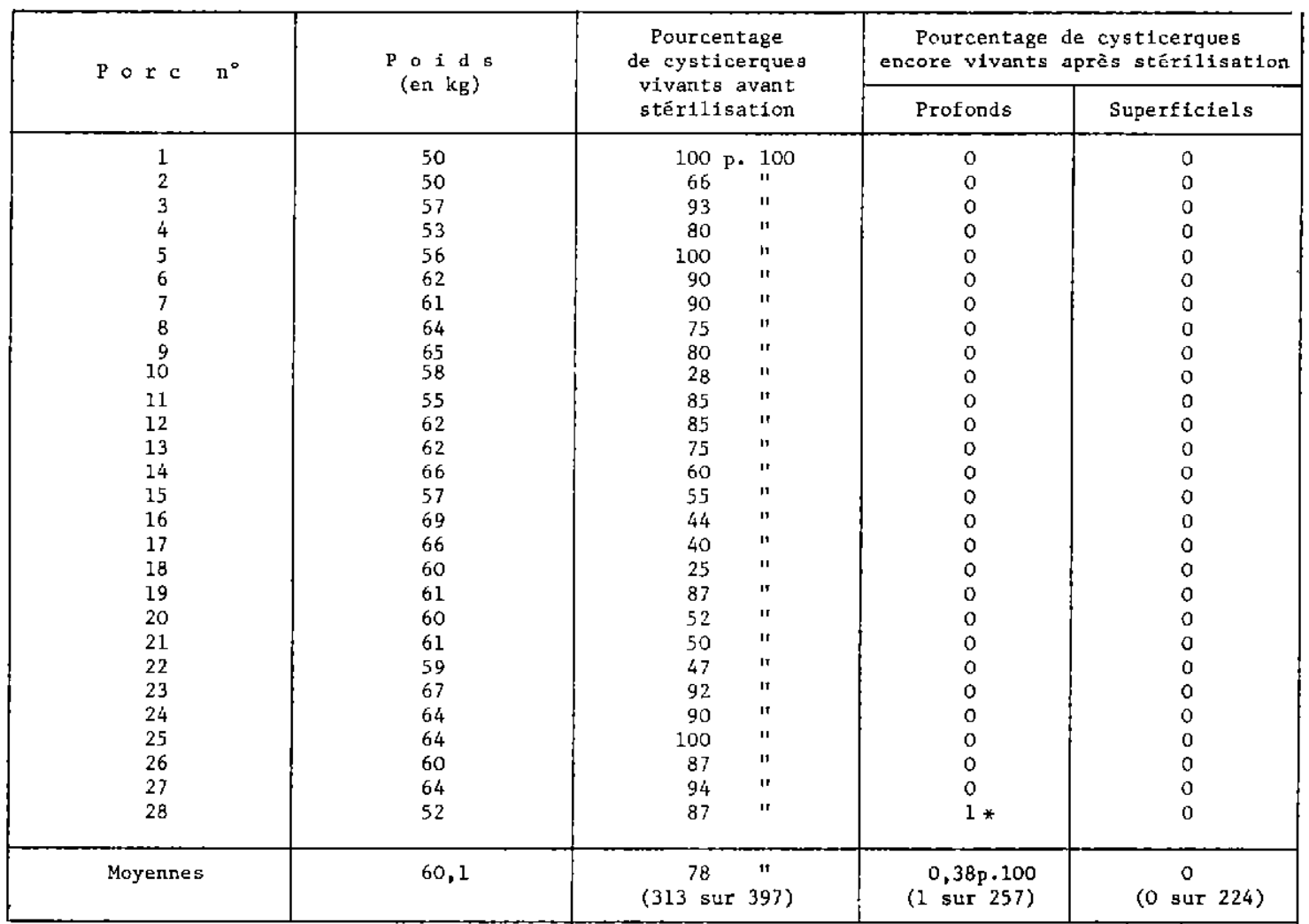

* S'évagine, mals meurt quelques minutes plus tard. 
TABLEAU N $N^{\circ} \mathrm{VII}$

Applications pratiques chez le zébu.

Stérillsation après entreposage durant 54 heures dans une ambiance de $-15^{\circ} \mathrm{C}$.

\begin{tabular}{|c|c|c|c|c|}
\hline \multirow{2}{*}{$\mathrm{Z}$ ë b u $\quad \mathrm{n}^{c}$} & \multirow{2}{*}{$\begin{array}{l}P \circ i d s \\
(e n k g)\end{array}$} & \multirow{2}{*}{$\begin{array}{l}\text { Pourcentage } \\
\text { de cysticerques } \\
\text { vivants avant } \\
\text { stêrilisation }\end{array}$} & \multicolumn{2}{|c|}{$\begin{array}{l}\text { Pourcentage de cysticerques } \\
\text { encore vivants après stêrilisation }\end{array}$} \\
\hline & & & Profonds & Superficiels \\
\hline $\begin{array}{r}1 \\
2 \\
3 \\
4 \\
5 \\
6 \\
7 \\
8 \\
9 \\
10 \\
11 \\
12 \\
13 \\
14\end{array}$ & $\begin{array}{c}150 \\
170 \\
214 \\
228 \\
117 \\
- \\
195 \\
190 \\
250 \\
- \\
215 \\
150 \\
180 \\
188\end{array}$ & $\begin{array}{rl}84 & \text { p. } 100 \\
88 & \text { " } \\
100 & " 1 \\
93 & " \\
100 & " \\
60 & " 1 \\
80 & " 1 \\
66 & " 1 \\
88 & " \\
100 & " \\
100 & " \\
100 & " \\
86 & " \\
100 & "\end{array}$ & $\begin{array}{l}0 \\
0 \\
0 \\
0 \\
0 \\
0 \\
- \\
0 \\
0 \\
0 \\
0 \\
0 \\
0 \\
0\end{array}$ & $\begin{array}{l}\overline{0} \\
0 \\
0 \\
- \\
- \\
- \\
\overline{0} \\
0 \\
- \\
- \\
- \\
-\end{array}$ \\
\hline Moyennes & 187,2 & $\begin{array}{l}87,9 \\
(212 \text { sur } 241)\end{array}$ & $\begin{array}{c}0 \\
\text { (o sur 161) }\end{array}$ & $\left(\begin{array}{c}0 \\
(0 \operatorname{sur} 51)\end{array}\right.$ \\
\hline
\end{tabular}

- $10^{\circ} \mathrm{C}$, plus de 34 heures. Par ailleurs, d'autres essais, réalisés sur 6 animaux, ont montré qu'à - $15^{\circ} \mathrm{C}$ durant 30 heures, les porcs ne sont pas tous assainis (5 sur 6).

En définitive, il a été décidé de conserver l'ambiance de $-15^{\circ} \mathrm{C}$ et de laisser au moins 35 heures les porcs ladres dans le tunnel de congélation.

\section{Applications pratiques}

Les résultats expérimentaux ont été vérifiés sur 28 porcs et 14 zébus massivement infestés (tableaux 6 et 7 ).

L'entreposage, dans une ambiance de $-15^{\circ} \mathrm{C}$, des carcasses de zébus durant 54 heures et des carcasses de porcs durant 35 heures assure pratiquement la destruction de tous les cysticerques: un seul d'entre eux, prélevé en profondeur chez un porc, s'est évaginé dans la bile à $39^{\circ} \mathrm{C}$, mais il n'a survécu que quelques minutes.

Ce système, en vigueur depuis 1966 à l'abattoir de Fort-Lamy, a donné jusqu'à présent toute satisfaction.

Dans les régions d'Afrique à élevage extensif, les avantages de la méthode sont évidents. La vitesse de rotation des carcasses à assainir se trouve accélérée et il n'est plus nécessaire de prévoir des installations importantes et oné- reuses. Il suffit d'adapter le volume de la chambre de congélation au nombre d'animaux ladres susceptibles d'être stérilisés dans l'année, en tenant compte du fait qu'il est théoriquement possible d'effectuer, chez le bcuf, de 9 à 10 opérations de congélation dans le mois, contre 3 à 5 par les procédés habituels.

Les différences observées, tant en ce qui concerne les températures que les délais nécessités pour les obtenir, sont certainement imputables au fait que les carcasses de ces divers animaux étaient sensiblement différentes tant en matière de poids, de volume, que d'état d'engraissement.

\section{LES COUTS}

Les coûts d'assainissement d'un kilogramme de viande ont été calculés en fonction du tonnage stérilisé, c'est-à-dire, pour l'année 1968 :

- 116 bcufs à 150 kilogrammes de moyenne, soit 17.400 kilogrammes.

- 109 porcs à 50 kilogrammes, soit 5.450 kilogrammes.

Trois postes de frais doivent être pris en considération :

1. Premier poste: 


\section{Entretien et amortissement}

\subsection{L'installation}

Installation frigorifique $\quad 740.000 \mathrm{~F} \mathrm{CFA}$ Génie civil . . . . . 240.000 F CFA Matériel de manutention . 92.000 F CFA

Total

1.072.000 F CFA

\subsection{Frais annuels}

Entretien 2 p. 100. 21.440 F CFA

Frigorifique 10 p. $100 . \quad 74.000$ F CFA

Génie civil 5 p. 100 . . . 12.000 F CFA

Manutention 10 p. 100

Total

5.200 F CFA

116.640 F CFA

1.3. Par kilogramme de viande

Porcs :

$\frac{116.640 \times 5.450}{22.950}=\frac{27.698}{5.450}=5,08 \mathrm{~F} \mathrm{CFA}$

Bæufs :

$\frac{116.640 \times 17.400}{22.950}=\frac{88.942}{17.400}=5,11 \mathrm{~F} \mathrm{CFA}$

2. Deuxième poste: Personnel

\subsection{Globalement}

Deux mancuvres à 200 F CFA par jour durant 365 jours :

$$
200 \times 2 \times 365=146.000 \text { F CFA }
$$

2.2. Par kilogramme de viande

Porcs :

$$
\frac{146.000 \times 5.450}{22.950}=\frac{34.671}{5.450}=6,36 \mathrm{~F} \mathrm{CFA}
$$

Bæufs :

$$
\frac{146.000 \times 17.400}{22.950}=\frac{111.329}{17.400}=6,39 \mathrm{~F} \mathrm{CFA}
$$

3. Troisième poste : Energie

\subsection{Frais globaux}

Puissance installée :

- 1 moteur électrique $5 \mathrm{ch}$,

- 1 ventilateur $1 \mathrm{ch}$, soit $6 \times 0,75=4,5 \mathrm{kVA}$.

L'installation électrique fonctionnant en automaticité, on peut estimer la consommation d'énergie à la moitié de ce qu'elle devrait être normalement :
Prix $\mathrm{kV}$ à Fort-Lamy : 22 F CFA, soit par heure $\frac{4,5 \times 22}{2}=50$ F CFA

3.2. Par kilogramme de viande

$$
\begin{aligned}
& \text { Porc : } \frac{50 \times 35}{750}=2,33 \text { F CFA } \\
& \text { Bœuf : } \frac{50 \times 54}{750}=3,60 \mathrm{~F} \mathrm{CFA}
\end{aligned}
$$

4. Si l'on additionne les chiffres figurant aux trois postes, un kilogramme de viande assainie revient à :

Porc :

$$
2,33+6,36+5,08=13,77 \text { F CFA }
$$

Bouf :

$$
3,60+6,35+5,11=15,10 \mathrm{~F} \mathrm{CFA}
$$

Pour mémoire, le prix moyen d'achat d'un kilogramme de carcasse est à Fort-Lamy de :

Pore : 200 F CFA

Bœuf : 50 à $60 \mathrm{~F} \mathrm{CFA}$

Pour éviter de trop pénaliser les viandes parasitées (le prix de la carcasse de bouf serait augmenté de $2.265 \mathrm{FCFA}$ et celui de la carcasse de porc de 688,5 F CFA), il serait souhaitable d'instaurer une taxe qui frapperait toutes les viandes, comme cela est d'ailleurs pratiqué à l'abattoir de Bangui. Pour 1968, compte tenu du fait qu'il a été tué 56.784 bœufs et 1.374 porcs, cette taxe aurait représenté :

Bœuf : $\frac{2.265 \times 116}{56.784}=4,62$ F CFA par tête Porc: $\frac{688,5 \times 109}{1.374}=54,61 \mathrm{~F} \mathrm{CFA}$ par tête ce qui est en définitive fort peu.

\section{CONCLUSIONS}

Des essais de stérilisation de viandes ladres ont été effectués à Fort-Lamy sur 45 porcs et 23 zébus originaires des zones sahélo-soudaniennes de la République du Tchad.

Les carcasses de zébus (187 kg en moyenne), préalablement réfrigérées, et celles de porcs (60 kg en moyenne) sont entreposées 54 heures dans le premier cas et 35 heures dans le second à la température de $-15^{\circ} \mathrm{C}$. 
Cette technique permet d'accélérer la vitesse de rotation des viandes dans le tunnel de congélation dont la capacité, par rapport aux installations conçues en fonction des normes habituelles $\left(-10^{\circ} \mathrm{C}\right.$ pendant 6 ou 10 jours) pourra être réduite, ce qui rend l'investissement de base moins onéreux.
Cependant, dans le cas d'un abattoir où l'installation ne fonctionne que quelques mois dans l'année en raison du faible nombre de carcasses à stériliser, les frais inhérents à cette opération finissent par être élevés. Ils devraient être couverts par une taxe frappant l'ensemble des animaux abattus.

\section{SUMMARY}

\section{Use of refrigeration for the sterilisation of measly meat at Fort-Lamy slaughter-house}

The authors worked on animal carcasses infected by cysticercosis, the livestock having originated from the Sahelo-Soudanian areas of Chad; the specimens examined were $187 \mathrm{~kg}$ zebu and $60 \mathrm{~kg}$ pig carcasses. It was found that complete sterilisation could be obtained by precooling at $5 \circ \mathrm{C}$ and storing at $-15^{\circ} \mathrm{C}$, zebu carcasses being stored for a period of $54 \mathrm{hrs}$ and pig carcasses for $35 \mathrm{hrs}$.

These tests have provided sterilisation standards, for infected carcasses, which can be applied to the greater part of regions in Africa where extensive breeding is carried out.

\section{RESUMEN}

\section{Utilización del frio para la esterilización de carnes cisticercosas en eI matadero frigorífico de Fort-Lamy}

Los autores efectuaron sus estudios sobre canales de animales atacados por cisticercosis, originarios de las regiones sahelo-sudanesas de Chad, teniendo los cebues un peso medio de $187 \mathrm{~kg}$ y los cerdos de $60 \mathrm{~kg}$. Obtuvieron su completa esterilización almacenandolas, después de refrigeración $\mathrm{a}+5^{\circ} \mathrm{C}$, a una temperatura de $-15^{\circ} \mathrm{C}$, durante 54 horas en lo concerniente a los cebues y 35 horas los cerdos.

Así determinan las normas de esterilización de las canales generalmente aplicables en la mayor parte de las regiones de Africa con ganaderia extensiva.

\section{BIBLIOGRAPHIE}

BIACI (F.), VELEZ (G.) et GUTIERREZ (M. L.), «Destrucción de los cisticercos en la carne de cerdo parasitada », Boln. Sanit. Pan. AM., 1965, 58 (4) : 303-07.

BARTELS (H.) et TANDLER (K.), \& Einige ergebnisse neuer untersuchungen über rinderfinnen ", Fleischwirtschaft, 1961, 13 : 904-13.

BARTELS (H), TANDLER (K.), « Die gefrier behandlung Schachfinniger rinderbei $-35^{\circ} \mathrm{C} »$, Fleischwirtschaft, 1963, $15: 87$.

BRAND (G.), «Vorschiften für die Fleischleschau», Köln, Deutsch. Gemsindeverlag GMBH, 1961, $116 \mathrm{p}$.

CLAREMBURG (A.), "Onderzockingen over de levensvatbaarheid van Cysticercus inermis $»$, Tiidschr. Diergeneesk., 1932, 59 (1) : 1-18.

EUZEBY (J.), « Les maladies vermineuses des animaux domestiques et leurs incidences sur la pathologie humaine. T. II. Maladies dues aux plathelminthes. Fasc. I. Cestodoses », Paris, Vigot frères, 1966, pp. $459-60$.
GINSBERG (A.) et collab., \& Bovine cysticercosis with particular reference to east Africa », Bull. Epizoor. Dis. Afr., 1956, 4 (1-2) : 27-39.

GRABER (M.), «La cysticercose bovine. Son importance dans les zones sahéliennes d'élevage de la République du Tchad », Rev. Elev. Méd. vét. Pays trop., 1959, 12 (2) : 121-48.

GRABER (M.) et THOME (M.), «La cysticercose bovine en République du Tchad », Rev. Elev. Méd. vét. Pays trop., 1964, 7 (3): 441-66.

GRABER (M.) et TABO (R.), «La cysticercose en milieu sédentaire et en milieu nomade ", Rev. Elev. Méd. vét. Pays trop., 1968, 21 (1) : 79-83.

GRABER (M.), * Helminthes et helminthiases. Bilan d'activité », Rapp. 1967, Lab. Farcha, t. III, 1968, pp. $70-4$,

KALLERT, " Het afsterven der runderfinnen bij het bevriezen van het vleesch », Z. Fl. Milchhyg., 1931, 41 (15): 319-20.

LACROUTS (M.), «Contribution à l'étude des améliorations que l'on doit attendre de l'utilisation du 
froid dans la commercialisation de la viande et du bétail dans les pays tropicaux $»$, Coll. Int. Abidjan, Bull. I.J.F., Annexe 1964 (3): 103-16.

LANDI (A.) et MONZINI (A.), «Osservazıoni sul comportamento e sulla vitalia del Cysticercus cellulosae alle basse temperature $»$, Clinica Vet. Milalano, 1954, 77 (9) : 264-8.

LAURENT (C.) et DEMARY (L.), \& L'abattoir frigorifique de Bangui », Coll. Int. Abidjan, 1964, Bull. I.I.F., Annexe 1964 (3): 117-23.

LEINATI (L.) et collab., " La elmintiasi dele uomo da alimenti di origine animale », Clinica Vet. Milano, 1963,$861 ; 173-217,242-57$ et $356-404$.

MALHEIRO (D. M.) et Collab., Observaçoes acérca da vitalidade do Cysticercus cellulosae quando submetido a bairas temperaturas ", Archos. Inst. Biol. S. Paulo, 1966, 33 (4) : 137-48.
MONZINI (A.) et LANDI (A.), «Osservazioni sul comportamento e sulla vitalia del Cysticercus bovis e del Cysticercus cellulosae alle basse temperature ", Atti III congr. Naz. Freddo, 1954, 421-7. NIEDERCHE (H.), "Stindiges einfrioren Schwachfinniger Rinder im Schnell Stühlraum bei - $16^{\circ} \mathrm{C}$, Schlacht - u. Viehhof - Zeit., 1964, 64: 151.

PAWEL (O.) et JANICEK (J.), « Moznote upravy uhrivého masa ionizyjicim zarenim », Vet. Med. Prague, 1963, 36 (2): 111-20.

Rapports Annuels de l'abattoir de Farcha. Statistiques 1963 à 1967.

VILJOEN (N.F.), Cysticercosis in swine and bovines, with special reference to South Africa conditions n, Onderstepoort J. vet. Res., 1937, 9 (2): 337-570. 